JOURNAL OF THE

AMERICAN MATHEMATICAL SOCIETY

Volume 26, Number 3, July 2013, Pages 831-852

S 0894-0347(2012)00741-3

Article electronically published on June 5, 2012

\title{
THE LOGARITHMIC MINKOWSKI PROBLEM
}

\author{
KÁROLY J. BÖRÖCZKY, ERWIN LUTWAK, DEANE YANG, AND GAOYONG ZHANG
}

\section{INTRODUCTION}

The setting for this paper is $n$-dimensional Euclidean space, $\mathbb{R}^{n}$. A convex body in $\mathbb{R}^{n}$ is a compact convex set that has a non-empty interior. A polytope in $\mathbb{R}^{n}$ is the convex hull of a finite set of points in $\mathbb{R}^{n}$ provided it has positive volume (i.e., $n$-dimensional volume). The convex hull of a subset of these points is called a face of the polytope if it lies entirely on the boundary of the polytope and if it has positive area (i.e., $(n-1)$-dimensional volume).

One of the cornerstones of the Brunn-Minkowski theory of convex bodies is the Minkowski problem. It can be stated in a simple way for polytopes:

Discrete Minkowski problem. Find necessary and sufficient conditions on a set of unit vectors $u_{1}, \ldots, u_{m}$ in $\mathbb{R}^{n}$ and a set of real numbers $a_{1}, \ldots, a_{m}>0$ that will guarantee the existence of an $m$-faced polytope in $\mathbb{R}^{n}$ whose faces have outer unit normals $u_{1}, \ldots, u_{m}$ and corresponding face-areas $a_{1}, \ldots, a_{m}$.

More than a century ago, this problem was completely solved by Minkowski himself 48: If the unit vectors do not lie on a closed hemisphere of $S^{n-1}$, then a solution to the Minkowski problem exists if and only if

$$
a_{1} u_{1}+\cdots+a_{m} u_{m}=0 .
$$

In addition, the solution is unique up to a translation.

The discrete Minkowski problem prescribes the areas of faces of a polytope. A natural, but still unsolved, problem involves prescribing the cone-volumes of the polytope. If a polytope contains the origin in its interior, then the cone-volume associated with a face of the polytope is the volume of the convex hull of the face and the origin.

Discrete logarithmic Minkowski problem. Find necessary and sufficient conditions on a set of unit vectors $u_{1}, \ldots, u_{m}$ in $\mathbb{R}^{n}$ and a set of real numbers $v_{1}, \ldots, v_{m}$ $>0$ that guarantee the existence of an $m$-faced polytope that contains the origin in its interior, whose faces have outer unit normals $u_{1}, \ldots, u_{m}$ and whose corresponding cone-volumes are $v_{1}, \ldots, v_{m}$.

Received by the editors September 18, 2011 and, in revised form, February 7, 2012.

2010 Mathematics Subject Classification. Primary 52A40.

Key words and phrases. Cone-volume measure, Minkowski problem, $L_{p}$-Minkowski problem, log-Minkowski problem.

The research of the first author was supported, in part, by EU FP7 IEF grant GEOSUMSET and OTKA 075016.

The research of the other three authors was supported, in part, by NSF Grant DMS-1007347. 
To state the logarithmic Minkowski problem for general convex bodies, we need to define the cone-volume measure of a convex body. If $K$ is a convex body in $\mathbb{R}^{n}$ that contains the origin in its interior, then the cone-volume measure, $V_{K}$, of $K$ is a Borel measure on the unit sphere $S^{n-1}$ defined for a Borel set $\omega \subset S^{n-1}$ by

$$
V_{K}(\omega)=\frac{1}{n} \int_{x \in \nu_{K}^{-1}(\omega)} x \cdot \nu_{K}(x) d \mathcal{H}^{n-1}(x),
$$

where $\nu_{K}: \partial^{\prime} K \rightarrow S^{n-1}$ is the Gauss map of $K$, defined on $\partial^{\prime} K$, the set of points of $\partial K$ that have a unique outer unit normal, and $\mathcal{H}^{n-1}$ is $(n-1)$-dimensional Hausdorff measure. In recent years, cone-volume measures have appeared in, e.g., [4], 18, 36, 37, 49, 50, 53, and [58.

If $K$ is a polytope whose outer unit normals are $u_{1}, \ldots, u_{m}$ and whose corresponding cone-volumes are $v_{1}, \ldots, v_{m}$, then the cone-volume measure of $K$ is the discrete measure

$$
V_{K}=v_{1} \delta_{u_{1}}+\cdots+v_{m} \delta_{u_{m}}
$$

where $\delta_{u_{i}}$ denotes the delta measure that is concentrated on $u_{i}$.

Logarithmic Minkowski problem. Find necessary and sufficient conditions on a finite Borel measure $\mu$ on the unit sphere $S^{n-1}$ so that $\mu$ is the cone-volume measure of a convex body in $\mathbb{R}^{n}$.

The associated partial differential equation for the logarithmic Minkowski problem is the following Monge-Ampère type equation on the unit sphere: For a given (data) function $f: S^{n-1} \rightarrow(0, \infty)$, solve

$$
h \operatorname{det}\left(h_{i j}+h \delta_{i j}\right)=f,
$$

where $h_{i j}$ is the covariant derivative of $h$ with respect to an orthonormal frame on $S^{n-1}$ and $\delta_{i j}$ is the Kronecker delta. Here the cone-volume measure $\mu$ is assumed to have the density $f$, with respect to spherical Lebesgue measure. In connection with (1.2), we observe a new phenomenon: the solution for measure data does not follow from the solution for function data, but requires a fundamentally new approach. This will be explained after the formulation of Theorem 1.1.

In [38, the second named author introduced the notion of $L_{p}$-surface area measure and posed the associated $L_{p}$-Minkowski problem which has the classical Minkowski problem and the logarithmic Minkowski problem as two important cases.

If $K$ is a convex body in $\mathbb{R}^{n}$ that contains the origin in its interior and $p \in \mathbb{R}$, then the $L_{p}$-surface area measure $S_{p}(K, \cdot)$ of $K$ is a Borel measure on the unit sphere $S^{n-1}$ defined on a Borel set $\omega \subset S^{n-1}$ by

$$
S_{p}(K, \omega)=\int_{x \in \nu_{K}^{-1}(\omega)}\left(x \cdot \nu_{K}(x)\right)^{1-p} d \mathcal{H}^{n-1}(x) .
$$

When $p=1$, the measure $S_{1}(K, \cdot)$ is the classical surface area measure of $K$. When $p=0$, the measure $\frac{1}{n} S_{0}(K, \cdot)$ is the cone-volume measure of $K$. When $p=2$, the measure $S_{2}(K, \cdot)$ is called the quadratic surface area measure of $K$, which was studied in [41, 42, and 35. Applications of the $L_{p}$-surface area measure to affine isoperimetric inequalities were given in, e.g., 6], 40, and 45]. 
The following $L_{p}$-Minkowski problem is one of the central problems in contemporary convex geometric analysis.

$L_{p}$-Minkowski problem. Find necessary and sufficient conditions on a finite Borel measure $\mu$ on the unit sphere $S^{n-1}$ so that $\mu$ is the $L_{p}$-surface area measure of a convex body in $\mathbb{R}^{n}$.

The case $p=1$ of the $L_{p}$-Minkowski problem is of course the classical Minkowski problem, which was solved by Minkowski, Alexandrov, and Fenchel and Jessen (see Schneider [55] for references). Landmark contributions to establishing regularity for the Minkowski problem are due to (among others) Lewy 34, Nirenberg [51, Cheng and Yau [8, Pogorelov [54, and Caffarelli [5].

For $p>1$, a solution to the $L_{p}$-Minkowski problem was given in 38] under the assumption that $p \neq n$ and that measure $\mu$ is even (assumes the same values on antipodal Borel subsets of $S^{n-1}$ ) . In [44, it was shown that, for $p \neq n$, the $L_{p^{-}}$ Minkowski problem has an equivalent volume-normalized formulation, and (using a slightly modified approach) a solution of the even volume-normalized $L_{p}$-Minkowski problem was given for all $p>1$. The regular even $L_{p}$-Minkowski problem was studied in [39. Extensions of the $L_{p}$-Minkowski problem are studied in [23].

In the plane $(n=2)$, the $L_{p}$-Minkowski problem was treated by Stancu [56, 57], Umanskiy [61, Chen [7, and by Jiang [31]. Solutions to the $L_{p}$-Minkowski problem are the homothetic solutions of Gauss curvature flows (see, e.g., [2], 3], 10], 14, [60]). When the measure $\mu$ is proportional to Lebesgue measure on the unit circle, $S^{1}$, solutions to the $L_{p}$-Minkowski problem in $\mathbb{R}^{2}$ are the homothetic solutions of isotropic curve flows classified by Andrews 3 .

The $L_{p}$-Minkowski problem (without the assumption that the data is even) was treated by Guan and Lin [21] and by Chou and Wang [9. Hug et al. 29] gave an alternate approach to some of the results of Chou and Wang $[9$.

The solution of the even $L_{p}$-Minkowski problem was a critical ingredient that allowed the authors of [43] to extend the affine Sobolev inequality [63] and obtain the $L_{p}$ affine Sobolev inequality [43, and later enabled Cianchi et al. [11] to establish the affine Moser-Trudinger and the affine Morrey-Sobolev inequalities. These were then strengthened by Haberl et al. in [24, 25, 26]. Connections of the $L_{p}$-Minkowski problem with optimal Sobolev norms were shown in [46].

There are other important works on extensions and analogues of the Minkowski problem; see, e.g., [12, 20], 22, 28, and 30].

Much of the past work on the $L_{p}$-Minkowski problem is limited to the case $p>1$. One of the reasons is that uniqueness for the $L_{p}$-Minkowski problem for $p>1$ can be shown by using mixed volume inequalities (see [38]). When $p<1$, the problem becomes challenging because there are no mixed volume inequalities available as of yet. The case $p=0$, called the logarithmic case, is probably the most important case with geometric significance because it is the singular case. The cone-volume measure is the only one among all the $L_{p}$-surface area measures (1.3) that is $\operatorname{SL}(n)$ invariant; i.e., for $\phi \in \mathrm{SL}(n)$,

$$
S_{0}\left(\phi^{t} K, \omega\right)=S_{0}(K,\langle\phi \omega\rangle),
$$

where $\langle\phi \omega\rangle=\{\phi u /|\phi u|: u \in \omega\}$. In light of the equivalence of the study of finitedimensional Banach spaces and that of origin-symmetric convex bodies and the fact that the cone-volume measure of an origin-symmetric convex body is even, the following even logarithmic Minkowski problem is of great interest. 
Even logarithmic Minkowski problem. Find necessary and sufficient conditions on an even Borel measure $\mu$ on the unit sphere $S^{n-1}$ so that $\mu$ is the cone-volume measure of an origin-symmetric convex body in $\mathbb{R}^{n}$.

It is the aim of this paper to solve the existence part of the even logarithmic Minkowski problem.

Definition. A finite Borel measure $\mu$ on $S^{n-1}$ is said to satisfy the subspace concentration inequality if, for every subspace $\xi$ of $\mathbb{R}^{n}$, such that $0<\operatorname{dim} \xi<n$,

$$
\mu\left(\xi \cap S^{n-1}\right) \leq \frac{1}{n} \mu\left(S^{n-1}\right) \operatorname{dim} \xi .
$$

The measure is said to satisfy the subspace concentration condition if in addition to satisfying the subspace concentration inequality (1.4), whenever

$$
\mu\left(\xi \cap S^{n-1}\right)=\frac{1}{n} \mu\left(S^{n-1}\right) \operatorname{dim} \xi,
$$

for some subspace $\xi$, then there exists a subspace $\xi^{\prime}$, which is complementary to $\xi$ in $\mathbb{R}^{n}$, so that also

$$
\mu\left(\xi^{\prime} \cap S^{n-1}\right)=\frac{1}{n} \mu\left(S^{n-1}\right) \operatorname{dim} \xi^{\prime},
$$

or equivalently so that $\mu$ is concentrated on $S^{n-1} \cap\left(\xi \cup \xi^{\prime}\right)$.

The measure $\mu$ on $S^{n-1}$ is said to satisfy the strict subspace concentration inequality if the inequality in (1.4) is strict for each subspace $\xi \subset \mathbb{R}^{n}$, such that $0<\operatorname{dim} \xi<n$.

The following theorem is the main result of this paper. It gives the necessary and sufficient conditions for the existence of a solution to the even logarithmic Minkowski problem.

Theorem 1.1. A non-zero finite even Borel measure on the unit sphere $S^{n-1}$ is the cone-volume measure of an origin-symmetric convex body in $\mathbb{R}^{n}$ if and only if it satisfies the subspace concentration condition.

We are dealing with the general case where the data is a measure (which of course includes as a special case the discrete log-Minkowski problem where the measure is concentrated on a finite set of points). This is much harder than the case where the given data is a function. It is remarkable that the subspace concentration condition, which is satisfied by all cone-volume measures of convex bodies, is also the critical and only condition that is needed for existence. For functions, the subspace concentration condition is trivially satisfied but for measures it is precisely what is necessary.

We need to observe a crucial difference between the log-Minkowski problem and the classical Minkowski problem, in fact, all the cases of the $L_{p}$-Minkowski problem where $p \geq 1$. Once the $L_{p}$-Minkowski problem has been solved (for any particular value of $p \geq 1$ ) for the case where the data consists of functions, the general $L_{p^{-}}$ Minkowski problem (where the data is given by measures) can be solved by an approximation argument. However, the solution to the log-Minkowski problem for the general case (i.e., for measures) does not follow from its solution for the cases where the data is given by functions, at least not by any approximation argument known to the authors.

The discrete planar case of Theorem 1.1 was proved by Stancu [56]. 
Uniqueness for the logarithmic Minkowski problem is not treated in this paper. In determining the ultimate shape of his worn stone, Firey 13 . showed that if the cone-volume measure of a smooth origin-symmetric convex body in $\mathbb{R}^{n}$ is a constant multiple of the Lebesgue measure on $S^{n-1}$, then the convex body must be a ball. Firey conjectured that his symmetry assumption was necessitated only by his methods. In $\mathbb{R}^{3}$, Firey's conjecture was established by Andrews [2].

Firey's theorem regarding unique determination by cone-volume measures can be shown to hold for measures other than Lebesgue measures. This will be treated in a separate paper.

\section{Preliminaries}

We develop some notation and, for quick later reference, list some basic facts about convex bodies. Good general references for the theory of convex bodies are provided by the books of Gardner [15], Gruber [19], Schneider [55], and Thompson [59].

The standard inner product of the vectors $x, y \in \mathbb{R}^{n}$ is denoted by $x \cdot y$. We write $|x|^{2}=x \cdot x$, and $S^{n-1}=\left\{x \in \mathbb{R}^{n}:|x|=1\right\}$ for the boundary of the Euclidean unit ball $B$ in $\mathbb{R}^{n}$. The volume of $B$ will be denoted by $\omega_{n}$.

The set of continuous functions on the sphere $S^{n-1}$ will be denoted by $C\left(S^{n-1}\right)$ and will always be viewed as equipped with the max-norm metric:

$$
|f-g|_{\infty}=\max _{u \in S^{n-1}}|f(u)-g(u)|,
$$

for $f, g \in C\left(S^{n-1}\right)$. The set of strictly positive continuous functions will be denoted by $C^{+}\left(S^{n-1}\right)$, and $C_{e}^{+}\left(S^{n-1}\right)$ will denote the subset of $C^{+}\left(S^{n-1}\right)$ consisting of only the even functions.

Write $V_{i}$ for the $i$-dimensional Lebesgue measure. When $i=n$, the subscript will be suppressed, and $V_{n}$ will be simply written as $V$. For $k$-dimensional Hausdorff measure, we write $\mathcal{H}^{k}$. The letter $\mu$ will be used exclusively to denote a finite Borel measure on $S^{n-1}$. For such a measure $\mu$, we denote by $|\mu|$ its total mass, i.e. $|\mu|=\mu\left(S^{n-1}\right)$.

We write $o$ for the origin of $\mathbb{R}^{n}$, and $\left[x_{1}, \ldots, x_{i}\right]$ to denote the convex hull of the points $x_{1}, \ldots, x_{i} \in \mathbb{R}^{n}$. For a non-zero $u \in \mathbb{R}^{n}$ or a linear subspace $\xi$, let $u^{\perp}$ and $\xi^{\perp}$ denote the orthogonal complement of the respective linear subspace. Moreover, write $\mathrm{P}_{\xi}: \mathbb{R}^{n} \rightarrow \xi$ for the orthogonal projection onto $\xi$.

A convex body is a compact convex subset of $\mathbb{R}^{n}$ with non-empty interior. The set of convex bodies in $\mathbb{R}^{n}$ containing the origin in their interiors is denoted by $\mathcal{K}_{o}^{n}$. The set of convex bodies in $\mathbb{R}^{n}$ that are symmetric about the origin will be denoted by $\mathcal{K}_{e}^{n}$. If $\xi \subset \mathbb{R}^{n}$ is an affine subspace, and $K$ is a convex body in $\xi$, then the set of relative interior points of $K$ with respect to $\xi$ is denoted by relint $K$.

The support function $h_{K}: \mathbb{R}^{n} \rightarrow \mathbb{R}$ of a compact, convex $K \subset \mathbb{R}^{n}$ is defined, for $x \in \mathbb{R}^{n}$, by

$$
h_{K}(x)=\max \{x \cdot y: y \in K\} .
$$

Note that support functions are positively homogeneous of degree one and subadditive. From the definition, it follows immediately that, for $\phi \in \operatorname{GL}(n)$, the support function of $\phi K=\{\phi x: x \in K\}$ is given by

$$
h_{\phi K}(x)=h_{K}\left(\phi^{t} x\right),
$$


for $x \in \mathbb{R}^{n}$. The support function of a body $K \in \mathcal{K}_{o}^{n}$ is strictly positive and continuous on the unit sphere $S^{n-1}$. If $x \in \partial^{\prime} K$, then the supporting distance of $\partial K$ at $x$ is defined to be $x \cdot \nu_{K}(x)=h_{K}\left(\nu_{K}(x)\right)$ and will be denoted by $d_{K}(x)$.

The set $\mathcal{K}_{o}^{n}$ will be viewed as equipped with the Hausdorff metric and thus for a sequence $\left\{K_{i}\right\}$ of bodies in $\mathcal{K}_{o}^{n}$ and a body $K \in \mathcal{K}_{o}^{n}$, we have $\lim _{i \rightarrow \infty} K_{i}=K$ provided that

$$
\left|h_{K_{i}}-h_{K}\right|_{\infty} \rightarrow 0 \text {. }
$$

A boundary point $x \in \partial K$ is said to have $u \in S^{n-1}$ as an outer normal provided $x \cdot u=h_{K}(u)$. A boundary point is said to be singular if it has more than one unit normal vector. It is well known (see, e.g., [55]) that the set of singular boundary points of a convex body has $\mathcal{H}^{n-1}$-measure equal to 0 .

For each Borel set $\omega \subset S^{n-1}$, the inverse spherical image of $\omega$ is the set of all points of $\partial K$ which have an outer unit normal belonging to the set $\omega$. Since the inverse spherical image of $\omega$ differs from $\nu_{K}^{-1}(\omega)$ by a set of $\mathcal{H}^{n-1}$-measure equal to 0 , we will often make no distinction between the two sets.

Associated with each convex body $K \in \mathcal{K}_{o}^{n}$ is a Borel measure $S_{K}$ on $S^{n-1}$ called the Aleksandrov-Fenchel-Jessen surface area measure or the surface area measure of $K$, defined for each Borel set $\omega \subset S^{n-1}$ as the $\mathcal{H}^{n-1}$-measure of the inverse spherical image of $\omega$, or equivalently

$$
S_{K}(\omega)=\mathcal{H}^{n-1}\left(\nu_{K}^{-1}(\omega)\right) .
$$

From (1.3) and (2.1), we see that $S_{1}(K, \cdot)=S_{K}$. We shall require the basic volume formula

$$
V(K)=\frac{1}{n} \int_{u \in S^{n-1}} h_{K}(u) d S_{K}(u) .
$$

As is well known, and easily shown, the measure $S_{p}(K, \cdot)$ is absolutely continuous with respect to the measure $S_{K}$, and its Radon-Nikodým derivative is $h_{K}^{1-p}$; i.e.,

$$
d S_{p}(K, \cdot)=h_{K}^{1-p} d S_{K} .
$$

Obviously, the measure $S_{p}(K, \cdot)$ is homogeneous of degree $n-p$ with respect to dilation of $K$, that is, $S_{p}(\lambda K, \cdot)=\lambda^{n-p} S_{p}(K, \cdot)$, for $\lambda>0$.

We will make use of the weak continuity of surface area measures; i.e., if $\left\{K_{i}\right\}$ is a sequence of bodies in $\mathcal{K}_{o}^{n}$, then

$$
\lim _{i \rightarrow \infty} K_{i}=K \in \mathcal{K}_{o}^{n} \Longrightarrow \lim _{i \rightarrow \infty} S_{K_{i}}=S_{K} \text {, weakly. }
$$

If $M$ is a convex body in $\mathbb{R}^{n}$ and $x_{0} \in M$, then $\rho_{-x_{0}+M}: \mathbb{R}^{n} \backslash\{0\} \rightarrow[0, \infty)$, the radial function of $M$ with respect to $x_{0}$, is defined for $x \in \mathbb{R}^{n} \backslash\{0\}$ by

$$
\rho_{-x_{0}+M}(x)=\max \left\{\lambda \geq 0: x_{0}+\lambda x \in M\right\} .
$$

Note that for $u \in S^{n-1}$, the distance from $x_{0}$ to $\partial M$ in direction $u$ is precisely $\rho_{-x_{0}+M}(u)$, that is, $x_{0}+\rho_{-x_{0}+M}(u) u \in \partial M$.

\section{THE VARIATIONAL METHOD}

A function $h \in C^{+}\left(S^{n-1}\right)$ defines a family $\left\{H_{u}\right\}_{u \in S^{n-1}}$ of hyperplanes

$$
H_{u}=\left\{x \in \mathbb{R}^{n}: x \cdot u=h(u)\right\} .
$$


Consider the intersection of the half-spaces that are associated to $h$ and bounded by the family $\left\{H_{u}\right\}_{u \in S^{n-1}}$. This gives rise to the convex body

$$
K=\bigcap_{u \in S^{n-1}}\left\{x \in \mathbb{R}^{n}: x \cdot u \leq h(u)\right\} .
$$

The body $K$ is called the Aleksandrov body (also known as the Wulff shape) associated with $h$. Note that since $h$ is both strictly positive and continuous its Aleksandrov body, $K$, must be an element of $\mathcal{K}_{o}^{n}$. The Aleksandrov body associated with $h$ can alternatively be defined as the unique maximal element, with respect to set inclusion, of the set

$$
\left\{Q \in \mathcal{K}_{o}^{n}: h_{Q} \leq h\right\} .
$$

Obviously, for the Aleksandrov body $K$ associated with the function $h$, we have

$$
h_{K} \leq h,
$$

and it turns out that, in fact,

$$
h_{K}=h, \quad \text { a.e. with respect to } S_{K} \text {. }
$$

If $h$ is the support function of a convex body $K \in \mathcal{K}_{o}^{n}$, then $K$ itself is the Aleksandrov body associated with $h$. If $h$ is an even function, then the Aleksandrov body associated with $h$ is origin-symmetric. We will need Aleksandrov's convergence lemma (see, e.g., [55, Lemma 6.5.2]): If the functions $h_{i} \in C^{+}\left(S^{n-1}\right)$ have associated Aleksandrov bodies $K_{i} \in \mathcal{K}_{o}^{n}$, then

$$
h_{i} \rightarrow h \in C^{+}\left(S^{n-1}\right) \quad \Longrightarrow \quad K_{i} \rightarrow K,
$$

where $K$ is the Aleksandrov body associated with $h$.

The volume $V(h)$ of a function $h \in C^{+}\left(S^{n-1}\right)$ is defined as the volume of the Aleksandrov body associated with $h$. Since the Aleksandrov body associated with the support function $h_{K}$ of a convex body $K \in \mathcal{K}_{o}^{n}$ is the body $K$ itself, we have

$$
V\left(h_{K}\right)=V(K) \text {. }
$$

Obviously, the functional $V: C^{+}\left(S^{n-1}\right) \rightarrow(0, \infty)$ is homogeneous of degree $n$; i.e., for $f \in C^{+}\left(S^{n-1}\right)$ and real $s>0$,

$$
V(s f)=s^{n} V(f) \text {. }
$$

From Aleksandrov's convergence lemma and the continuity of ordinary volume on $\mathcal{K}_{o}^{n}$ we see that

$$
V: C^{+}\left(S^{n-1}\right) \rightarrow(0, \infty) \text { is continuous. }
$$

Let $I \subset \mathbb{R}$ be an interval containing 0 and $h_{t}(u)=h(t, u): I \times S^{n-1} \rightarrow(0, \infty)$ be continuous. For fixed $t \in I$, let

$$
K_{t}=\bigcap_{u \in S^{n-1}}\left\{x \in \mathbb{R}^{n}: x \cdot u \leq h_{t}(u)\right\}
$$

be the Aleksandrov body associated with $h_{t}$. The family of convex bodies $K_{t}$ will be called the family of Aleksandrov bodies associated with $h_{t}$. Obviously, we can rewrite (3.1) as

$$
h_{K_{t}} \leq h_{t} \quad \text { and } \quad h_{K_{t}}=h_{t} \text {, a.e. with respect to } S_{K_{t}},
$$

for each $t \in I$. 
The following form (proved in, e.g., [23]) of Aleksandrov's Lemma (see, e.g., [55]) will be needed.

Lemma 3.1. Suppose $I \subset \mathbb{R}$ is an open interval containing 0 and that the function $h_{t}=h(t, u): I \times S^{n-1} \rightarrow(0, \infty)$ is continuous. If, as $t \rightarrow 0$, the convergence in

$$
\frac{h_{t}-h_{0}}{t} \rightarrow f=\left.\frac{\partial h_{t}}{\partial t}\right|_{t=0}
$$

is uniform on $S^{n-1}$, and if $K_{t}$ denotes the Aleksandrov body associated with $h_{t}$, then

$$
\lim _{t \rightarrow 0} \frac{V\left(K_{t}\right)-V\left(K_{0}\right)}{t}=\int_{S^{n-1}} f d S_{K_{0}}
$$

\section{A minimization PROBlem}

Let $\mu$ be a finite even Borel measure on $S^{n-1}$ with total mass $|\mu|>0$. Define the logarithmic functional $\Phi_{\mu}: \mathcal{K}_{e}^{n} \rightarrow \mathbb{R}$ by

$$
\Phi_{\mu}(K)=\int_{S^{n-1}} \log h_{K} d \mu .
$$

Consider the minimization problem,

$$
\inf \left\{\Phi_{\mu}(Q): V(Q)=|\mu| \text { and } Q \in \mathcal{K}_{e}^{n}\right\} .
$$

Lemma 4.1. Let $\mu$ be a finite even Borel measure on $S^{n-1}$ with $|\mu|>0$. If $K_{0} \in \mathcal{K}_{e}^{n}$ is an origin-symmetric convex body such that $V\left(K_{0}\right)=|\mu|$ and

$$
\Phi_{\mu}\left(K_{0}\right)=\inf \left\{\Phi_{\mu}(Q): V(Q)=|\mu| \text { and } Q \in \mathcal{K}_{e}^{n}\right\},
$$

then the measure $\mu$ is the cone-volume measure of $K_{0}$.

Proof. It is easily seen that it is sufficient to establish the lemma under the assumption that $\mu$ is a probability measure.

Define the functional $M_{0}: C_{e}^{+}\left(S^{n-1}\right) \rightarrow(0, \infty)$ by

$$
M_{0}(q)=\frac{1}{V(q)^{1 / n}} \exp \left(\int_{S^{n-1}} \log q d \mu\right)
$$

for $q \in C_{e}^{+}\left(S^{n-1}\right)$. Since the functional $V: C_{e}^{+}\left(S^{n-1}\right) \rightarrow(0, \infty)$ is continuous, we see that the functional $M_{0}: C_{e}^{+}\left(S^{n-1}\right) \rightarrow(0, \infty)$ is continuous as well. From (3.3) we see that the functional $M_{0}: C_{e}^{+}\left(S^{n-1}\right) \rightarrow(0, \infty)$ is homogeneous of degree 0 ; i.e., for $q \in C_{e}^{+}\left(S^{n-1}\right)$ and real $s>0$, we have $M_{0}(s q)=M_{0}(q)$.

Consider the minimization problem,

$$
\inf \left\{M_{0}(q): q \in C_{e}^{+}\left(S^{n-1}\right)\right\} .
$$

Suppose $f \in C_{e}^{+}\left(S^{n-1}\right)$. Let $K$ be the Aleksandrov body associated with $f$. Then $V(f)=V\left(h_{K}\right)=V(K)$ but $h_{K} \leq f$. Therefore, $M_{0}\left(h_{K}\right) \leq M_{0}(f)$. We can therefore limit our search for the infimum of $M_{0}$ by restricting our attention to support functions of origin-symmetric convex bodies. Since $M_{0}$ is homogeneous of degree 0 , the infimum of $M_{0}$ is

$$
\inf \left\{M_{0}(q): q \in C_{e}^{+}\left(S^{n-1}\right)\right\}=\inf \left\{e^{\Phi_{\mu}(Q)}: V(Q)=1 \text { and } Q \in \mathcal{K}_{e}^{n}\right\} .
$$


The hypothesis of our lemma is that the right infimum is in fact a minimum and that it is attained at $K_{0} \in \mathcal{K}_{e}^{n}$. Therefore, the support function $h_{K_{0}}>0$ is a solution of the minimization problem (4.3); i.e.,

$$
\inf \left\{M_{0}(q): q \in C_{e}^{+}\left(S^{n-1}\right)\right\}=M_{0}\left(h_{K_{0}}\right) .
$$

Suppose $g \in C_{e}\left(S^{n-1}\right)$ is arbitrary but fixed. Consider the family $h_{t} \in C_{e}^{+}\left(S^{n-1}\right)$, where the function $h_{t}=h(t, \cdot): \mathbb{R} \times S^{n-1} \rightarrow(0, \infty)$ is defined by

$$
h_{t}=h(t, \cdot)=h_{K_{0}} e^{t g},
$$

and let $K_{t}$ denote the Aleksandrov body associated with $h_{t}$ (since $h_{0}$ is the support function of the convex body $K_{0}$, our notation is consistent).

Since $g$ is bounded on $S^{n-1}$, for $h_{t}=h_{K_{0}} e^{t g}$, the hypothesis of Lemma 3.1.

$$
\frac{h_{t}-h_{0}}{t} \rightarrow g h_{K_{0}}, \quad \text { uniformly on } S^{n-1},
$$

as $t \rightarrow 0$, is satisfied and we get from Lemma 3.1

$$
\left.\frac{d}{d t} V\left(K_{t}\right)\right|_{t=0}=\int_{S^{n-1}} g h_{K_{0}} d S_{K_{0}} .
$$

Now (4.5) shows that the function $t \mapsto M_{0}\left(h_{t}\right)$, where

$$
M_{0}\left(h_{t}\right)=V\left(K_{t}\right)^{-1 / n} \exp \left(\int_{S^{n-1}} \log \left(h_{K_{0}} e^{t g}\right) d \mu\right),
$$

is differentiable at $t=0$, and, after recalling that by hypothesis $V\left(K_{0}\right)=1$, and using (4.5), differentiating in (4.6) gives us

$$
\left.\frac{d}{d t} M_{0}\left(h_{t}\right)\right|_{t=0}=\left[-\frac{1}{n} \int_{S^{n-1}} g h_{K_{0}} d S_{K_{0}}+\int_{S^{n-1}} g d \mu\right] \exp \left(\int_{S^{n-1}} \log h_{K_{0}} d \mu\right) .
$$

But (4.4) shows that the function $t \mapsto M_{0}\left(h_{t}\right)$ has a minimum at $t=0$ which gives

$$
\left.\frac{d}{d t} M_{0}\left(h_{t}\right)\right|_{t=0}=0
$$

and now (4.7) allows us to conclude that

$$
\frac{1}{n} \int_{S^{n-1}} g h_{K_{0}} d S_{K_{0}}=\int_{S^{n-1}} g d \mu .
$$

But since this must hold for arbitrary $g \in C_{e}\left(S^{n-1}\right)$, we conclude that

$$
d \mu=\frac{1}{n} h_{K_{0}} d S_{K_{0}}
$$

as desired.

\section{NeCESSARY CONDITIONS FOR EXISTENCE}

We shall use the method of symmetrization to prove the necessity part of Theorem 1.1. Consider a proper subspace $\xi \subset \mathbb{R}^{n}$ and let $m=\operatorname{dim} \xi$. We consider the symmetrization of the convex body $K \in \mathcal{K}_{o}^{n}$ with respect to $\xi$. For each $x \in \mathrm{P}_{\xi} K$, we replace $K \cap\left(x+\xi^{\perp}\right)$ with the $(n-m)$-dimensional ball $B_{r(x)}^{n-m}(x)$ in $x+\xi^{\perp}$ 
that is centered at $x$ and whose radius $r(x)$ is chosen so that $\omega_{n-m} r(x)^{n-m}=$ $V_{n-m}\left(K \cap\left(x+\xi^{\perp}\right)\right)$; i.e., $r(x)$ is chosen so that $B_{r(x)}^{n-m}(x)$ has the same $(n-m)$ dimensional volume as $K \cap\left(x+\xi^{\perp}\right)$. Denote the new body by $\mathrm{S}_{\xi} K$. That is,

$$
\mathrm{S}_{\xi} K=\bigcup_{x \in \mathrm{P}_{\xi} K} B_{r(x)}^{n-m}(x), \quad \omega_{n-m} r(x)^{n-m}=V_{n-m}\left(K \cap\left(x+\xi^{\perp}\right)\right) .
$$

As is well known (see, e.g., [17]), the Brunn-Minkowski inequality (see the beautiful survey [16) implies that $\mathrm{S}_{\xi} K$ is also a convex body. By Fubini's theorem, in fact, by Cavalieri's principle, $V(K)=V\left(\mathrm{~S}_{\xi} K\right)$. If $K$ is origin-symmetric, then $\mathrm{S}_{\xi} K$ is also origin-symmetric.

For notational simplicity, denote $\mathrm{S}_{\xi} K$ by $\tilde{K}$, and denote the Gauss map of $K$ by $\nu$ and that of $\tilde{K}$ by $\tilde{\nu}$. Also abbreviate $B_{r(x)}^{n-m}(x)$ by $B(x)$. If $C \subset \mathbb{R}^{n}$ is convex and compact, then for notational simplicity, write $\partial C$ for the relative boundary of $C$, with respect to the affine hull of $C$.

Lemma 5.1. Suppose $\xi \subset \mathbb{R}^{n}$ is a subspace of $\mathbb{R}^{n}$ such that $0<\operatorname{dim} \xi<n$, and suppose $K \in \mathcal{K}_{o}^{n}$. Then the cone-volume measures of $K$ and the symmetrization, $\tilde{K}$, of $K$ about $\xi$ satisfy

$$
V_{\tilde{K}}\left(\xi \cap S^{n-1}\right)=V_{K}\left(\xi \cap S^{n-1}\right) .
$$

Proof. Let $m=\operatorname{dim} \xi$ and let $S^{m-1}=\xi \cap S^{n-1}$.

For $z \in \mathbb{R}^{n}$, write $z=(x, y)$, where $x=\mathrm{P}_{\xi} z$ and $y=\mathrm{P}_{\xi} z$. We will identify $x$ with $(x, 0)$. If $z \in \nu^{-1}\left(S^{m-1}\right)$, then $x \in \partial\left(\mathrm{P}_{\xi} K\right)$. But for $x \in \partial\left(\mathrm{P}_{\xi} K\right)$ and $u \in S^{m-1}=\xi \cap S^{n-1}$, we obviously have $z \cdot u=x \cdot u$ because $z \in x+\xi^{\perp}$. This shows that $z \cdot \nu(z)$ is independent of $y \in K \cap\left(x+\xi^{\perp}\right)$ for $z \in \nu^{-1}\left(S^{m-1}\right)$. But $z \cdot \nu(z)$ is the supporting distance of $\partial K$ at $z$ as well as $d_{\mathrm{P}_{\xi} K}(x)$, the supporting distance of $\partial\left(\mathrm{P}_{\xi} K\right)$ at $x$ for $\mathcal{H}^{m-1}$-almost all $x$ on $\partial\left(\mathrm{P}_{\xi} K\right)$. Similarly, for $z \in \tilde{\nu}^{-1}\left(S^{m-1}\right)$, we also have $z \cdot \tilde{\nu}(z)=d_{\mathrm{P}_{\xi} \tilde{K}}(x)=d_{\mathrm{P}_{\xi} K}(x)$ because $\mathrm{P}_{\xi} K=\mathrm{P}_{\xi} \tilde{K}$. This, together with $V_{n-m}(B(x))=V_{n-m}\left(K \cap\left(x+\xi^{\perp}\right)\right)$ and $\mathrm{P}_{\xi} K=\mathrm{P}_{\xi} \tilde{K}$, gives

$$
\begin{aligned}
V_{K}\left(S^{m-1}\right) & =\frac{1}{n} \int_{z \in \nu^{-1}\left(S^{m-1}\right)} z \cdot \nu(z) d \mathcal{H}^{n-1}(z) \\
& =\frac{1}{n} \int_{x \in \partial\left(\mathrm{P}_{\xi} K\right)} d_{\mathrm{P}_{\xi} K}(x) V_{n-m}\left(K \cap\left(x+\xi^{\perp}\right)\right) d \mathcal{H}^{m-1}(x) \\
& =\frac{1}{n} \int_{x \in \partial\left(\mathrm{P}_{\xi} \tilde{K}\right)} d_{\mathrm{P}_{\xi} \tilde{K}}(x) V_{n-m}(B(x)) d \mathcal{H}^{m-1}(x) \\
& =\frac{1}{n} \int_{z \in \tilde{\nu}^{-1}\left(S^{m-1}\right)} z \cdot \tilde{\nu}(z) d \mathcal{H}^{n-1}(z) \\
& =V_{\tilde{K}}\left(S^{m-1}\right) .
\end{aligned}
$$

The necessity part of Theorem 1.1 is presented in Theorem 5.2 below. For origin-symmetric polytopes, the subspace concentration inequality of Theorem 5.2 was established previously by He, Leng and Li [27, with an alternate proof given by Xiong [62. We now give a new proof that is valid for arbitrary origin-symmetric convex bodies. Applications of this theorem to reverse affine isoperimetric inequalities were given in [27] and 62]. 
Theorem 5.2. The cone-volume measure of an origin-symmetric convex body in $\mathbb{R}^{n}$ satisfies the subspace concentration condition.

Proof. Suppose $K$ is an origin-symmetric convex body in $\mathbb{R}^{n}$. Let $\xi \subset \mathbb{R}^{n}$ be a subspace such that $0<\operatorname{dim} \xi=m<n$, and let $S^{m-1}=\xi \cap S^{n-1}$. Let $\tilde{K}$ be the symmetrization of $K$ with respect to $\xi$.

For $y \in B(o)=\mathrm{P}_{\xi^{\perp}} \tilde{K}$, let $\rho_{-y+\tilde{K}}=\rho_{\xi \cap(-y+\tilde{K})}: S^{m-1} \rightarrow(0, \infty)$, be the radial function of $\xi \cap(-y+\tilde{K})$.

There are three basic observations here. First, for each $u \in S^{m-1}$, the value of $\rho_{-y+\tilde{K}}(u)$ is independent of $y \in \mathrm{P}_{\xi^{\perp}} B\left(\rho_{\tilde{K}}(u) u\right)$. Then, that for each $z=(x, y) \in$ $\tilde{\nu}^{-1}\left(S^{m-1}\right)$, the value of $z \cdot \tilde{\nu}(z)$ is independent of $y \in\left(x+\xi^{\perp}\right) \cap \tilde{K}=B(x)$. Finally, $z \cdot \tilde{\nu}(z)$, the supporting distance of $\partial \tilde{K}$ at $z$, is equal to $d_{\mathrm{P}_{\xi} \tilde{K}}(x)$, the supporting distance of $\partial\left(\mathrm{P}_{\xi} \tilde{K}\right)$ at $x$, for $\mathcal{H}^{m-1}$-almost all $x$ on $\partial\left(\mathrm{P}_{\xi} \tilde{K}\right)$. From this and the definition of cone-volume measure we have

$$
\begin{aligned}
V_{\tilde{K}}\left(S^{m-1}\right) & =\frac{1}{n} \int_{z \in \tilde{\nu}^{-1}\left(S^{m-1}\right)} z \cdot \tilde{\nu}(z) d \mathcal{H}^{n-1}(z) \\
& =\frac{1}{n} \int_{x \in \partial\left(\mathrm{P}_{\xi} \tilde{K}\right)} d_{\mathrm{P}_{\xi} \tilde{K}}(x) V_{n-m}(B(x)) d \mathcal{H}^{m-1}(x) \\
& =\frac{1}{n} \int_{u \in S^{m-1}} \rho_{\tilde{K}}(u)^{m} V_{n-m}\left(B\left(\rho_{\tilde{K}}(u) u\right)\right) d \mathcal{H}^{m-1}(u) \\
& =\frac{1}{n} \int_{u \in S^{m-1}}\left(\int_{y \in \mathrm{P}_{\xi^{\perp}} B\left(\rho_{\tilde{K}}(u) u\right)} \rho_{-y+\tilde{K}}(u)^{m} d \mathcal{H}^{n-m}(y)\right) d \mathcal{H}^{m-1}(u),
\end{aligned}
$$

where in going from the second to the third line we changed variables $x=\rho_{\tilde{K}}(u) u$, for $u \in S^{m-1}$, and used the fact that

$$
d_{\mathrm{P}_{\xi} \tilde{K}}(x) d \mathcal{H}^{m-1}(x)=\rho_{\tilde{K}}(u)^{m} d \mathcal{H}^{m-1}(u)
$$

(see, e.g., [42, Lemma 2).

Since $B(o)$ is maximal, $\tilde{K}$ is obviously the (disjoint) union of the fibers $(y+\xi) \cap \tilde{K}$, with $y \in B(o)$. Thus,

$$
\begin{aligned}
V(\tilde{K}) & =\int_{y \in B(o)} V_{m}((y+\xi) \cap \tilde{K}) d \mathcal{H}^{n-m}(y) \\
& =\int_{y \in B(o)} V_{m}(\xi \cap(-y+\tilde{K})) d \mathcal{H}^{n-m}(y) \\
& =\frac{1}{m} \int_{y \in B(o)} \int_{u \in S^{m-1}} \rho_{-y+\tilde{K}}(u)^{m} d \mathcal{H}^{m-1}(u) d \mathcal{H}^{n-m}(y) .
\end{aligned}
$$

Since $K$ is origin-symmetric, the ball $B(x)$ attains its maximum size when $x=o$. Thus, $\mathrm{P}_{\xi^{\perp}} B\left(\rho_{\tilde{K}}(u) u\right) \subseteq B(o)$, and we get

$$
V_{\tilde{K}}\left(S^{m-1}\right) \leq \frac{m}{n} V(\tilde{K})
$$

with equality if and only if $\mathrm{P}_{\xi^{\perp}} B\left(\rho_{\tilde{K}}(u) u\right)=B(o)$, for all $u \in S^{m-1}$. Since $V(K)=$ $V(\tilde{K})$, Lemma 5.1 now allows us to conclude that

$$
V_{K}\left(\xi \cap S^{n-1}\right) \leq \frac{m}{n} V_{K}\left(S^{n-1}\right) ;
$$


that is, the subspace concentration inequality holds for the cone-volume measure of $K$.

To show that the cone-volume measure satisfies the subspace concentration condition, we now analyze the implications of equality in (5.1). To that end, assume that there is equality in (5.1). Then $\mathrm{P}_{\xi \perp} B\left(\rho_{\tilde{K}}(u) u\right)=B(o)$, for all $u \in S^{m-1}$. Suppose $x \in \mathrm{P}_{\xi} K \backslash\{o\}$. The Brunn-Minkowski inequality yields $\mathrm{P}_{\xi} B\left(\rho_{\tilde{K}}(u) u\right) \subset$ $\mathrm{P}_{\xi^{\perp}} B(x) \subset B(o)$ for $u=x /|x|$. Therefore, $\mathrm{P}_{\xi^{\perp}} B(x)=B(o)$ for each $x \in \mathrm{P}_{\xi} K$, and hence $V_{n-m}\left(\left(x+\xi^{\perp}\right) \cap K\right)$ is independent of $x \in \mathrm{P}_{\xi} K$. The equality conditions of the Brunn-Minkowski inequality tell us that this can only happen when $\left(x+\xi^{\perp}\right) \cap K$ is a translate of $\xi^{\perp} \cap K$ for each $x \in \mathrm{P}_{\xi} K$.

Since $K$ is origin-symmetric, so is $\xi^{\perp} \cap K$. Thus, for each $x \in \mathrm{P}_{\xi} K$ the body $\left(x+\xi^{\perp}\right) \cap K$ (being just a translate of $\left.\xi^{\perp} \cap K\right)$ has a center of symmetry. As $x$ traverses a line segment in $\mathrm{P}_{\xi} K$, the convexity of $K$ guarantees that all the boundary points of $\left(x+\xi^{\perp}\right) \cap K$ traverse parallel line segments, and thus the center of $\left(x+\xi^{\perp}\right) \cap K$ also traverses a parallel line segment as well. Therefore, as $x$ varies in $\mathrm{P}_{\xi} K$, the center of $\left(x+\xi^{\perp}\right) \cap K$ lies in an $m$-dimensional origin-symmetric convex compact set, which is

$$
C=\left\{z \in K: z+\left(\xi^{\perp} \cap K\right) \subset K\right\} .
$$

Thus

$$
K=\left(\xi^{\perp} \cap K\right)+C,
$$

which shows that we can write $K$ as the Minkowski sum of an $(n-m)$-dimensional and an $m$-dimensional convex set.

Let $\xi^{\prime}$ be the orthogonal complement of the linear hull of $C$. Now,

$$
\partial K \subseteq\left(\partial\left(\xi^{\perp} \cap K\right)+\operatorname{relint} C\right) \cup\left(\operatorname{relint}\left(\xi^{\perp} \cap K\right)+\partial C\right) \cup\left(\partial\left(\xi^{\perp} \cap K\right)+\partial C\right) .
$$

But $\nu\left(\partial\left(\xi^{\perp} \cap K\right)+\right.$ relint $\left.C\right) \subset S^{n-1} \cap \xi^{\prime}$ and $\nu\left(\operatorname{relint}\left(\xi^{\perp} \cap K\right)+\partial C\right) \subset S^{n-1} \cap \xi$, while $\mathcal{H}^{n-1}\left(\partial\left(\xi^{\perp} \cap K\right)+\partial C\right)=0$. Therefore, the cone-volume measure $V_{K}$ is concentrated on $S^{n-1} \cap\left(\xi \cup \xi^{\prime}\right)$.

\section{Minimizing the Logarithmic Functional}

In this section, we prove that the necessity condition for cone-volume measures is sufficient to imply the existence of a solution of the minimization problem (4.2).

We shall require the following trivial fact:

Lemma 6.1. If real $\alpha_{1}, \ldots, \alpha_{n} \geq 0$ satisfy

$$
\frac{\alpha_{1}+\cdots+\alpha_{i}}{i}<\frac{1}{n}, \quad \text { for all } i=1, \ldots, n-1,
$$

while

$$
\alpha_{1}+\cdots+\alpha_{n}=1
$$

then there exists $t \in(0,1]$ such that for $\lambda=(1-t) / n$, and

$$
\beta_{i}=\alpha_{i}-\lambda, \text { for all } i=1, \ldots, n-1, \text { while } \beta_{n}=\alpha_{n}-\lambda-t,
$$

we have

$$
\beta_{1}+\cdots+\beta_{i} \leq 0, \quad \text { for all } i=1, \ldots, n-1,
$$

and

$$
\beta_{1}+\cdots+\beta_{n}=0
$$


Proof. Choose an $i_{o} \in\{1, \ldots, n-1\}$ such that

$$
\frac{\alpha_{1}+\cdots+\alpha_{i}}{i} \leq \frac{\alpha_{1}+\cdots+\alpha_{i_{o}}}{i_{o}} \quad \text { for all } i=1, \ldots, n-1 .
$$

From (6.1) we see that there exists a $t \in(0,1]$ such that

$$
\begin{aligned}
\frac{\alpha_{1}+\cdots+\alpha_{i_{o}}}{i_{o}} & =(1-t) \frac{1}{n}=\lambda, \\
\frac{\alpha_{1}+\cdots+\alpha_{i}}{i} & \leq(1-t) \frac{1}{n}=\lambda, \quad \text { for all } i=1, \ldots, n-1 .
\end{aligned}
$$

Then

$$
\begin{aligned}
& \beta_{1}+\cdots+\beta_{i}=\alpha_{1}+\cdots+\alpha_{i}-i \lambda \leq 0, \quad \text { for all } i=1, \ldots, n-1, \\
& \beta_{1}+\cdots+\beta_{n}=\alpha_{1}+\cdots+\alpha_{n}-n \lambda-t=1-n \lambda-t=0 .
\end{aligned}
$$

Lemma 6.2. Suppose $\mu$ is a probability measure on $S^{n-1}$ that satisfies the strict subspace concentration inequality. For each positive integer $l$, let $u_{1, l}, \ldots, u_{n, l}$ be an orthonormal basis of $\mathbb{R}^{n}$, and suppose that $h_{1, l}, \ldots, h_{n, l}$ are $n$ sequences of positive real numbers such that $h_{1, l} \leq \cdots \leq h_{n, l}$, and such that the product $h_{1, l} \cdots h_{n, l} \geq 1$, and $\lim _{l \rightarrow \infty} h_{n, l}=\infty$. Then, for the cross-polytopes $Q_{l}=\left[ \pm h_{1, l} u_{1, l}, \ldots, \pm h_{n, l} u_{n, l}\right]$, the sequence

$$
\Phi_{\mu}\left(Q_{l}\right)=\int_{S^{n-1}} \log h_{Q_{l}} d \mu
$$

is not bounded from above.

Proof. For each $l$, we obviously have

$$
h_{Q_{l}}(v)=\max _{1 \leq i \leq n} h_{i, l}\left|v \cdot u_{i, l}\right|,
$$

for $v \in S^{n-1}$. After taking suitable subsequences, we may conclude the existence of an orthonormal basis $u_{1}, \ldots, u_{n}$ of $\mathbb{R}^{n}$ with

$$
\lim _{l \rightarrow \infty} u_{i, l}=u_{i} \in S^{n-1} \quad \text { for all } i=1, \ldots, n .
$$

For $i=1, \ldots, n$, let $\xi_{i}$ be the subspace spanned by $\left\{u_{1}, \ldots, u_{i}\right\}$, and for convenience let $\xi_{0}=\{o\}$. Since $\mu$ is a probability measure that satisfies the strict subspace concentration inequality, we have

$$
\sum_{j=1}^{i} \mu\left(S^{n-1} \cap\left(\xi_{j} \backslash \xi_{j-1}\right)\right)=\mu\left(S^{n-1} \cap \xi_{i}\right)<\frac{i}{n},
$$

for $i=1, \ldots, n-1$.

Next we observe that for each $v \in S^{n-1}$, there exists a $u_{i}$ such that $\left|v \cdot u_{i}\right| \geq 1 / \sqrt{n}$. This simple observation can be used to easily see that for each $\eta \in(0,1 / \sqrt{n})$, the sets

$$
A_{i, \eta}=\left\{v \in S^{n-1}:\left|v \cdot u_{i}\right| \geq \eta \text { and }\left|v \cdot u_{j}\right|<\eta \text { for } j>i\right\}, \quad i=1, \ldots, n,
$$

form a disjoint partition of $S^{n-1}$. Define

$$
B_{i, \eta}=\left\{v \in S^{n-1}:\left|v \cdot u_{i}\right|>0 \text { and }\left|v \cdot u_{j}\right|<\eta \text { for } j>i\right\}, \quad i=1, \ldots, n .
$$

We claim that

$$
\lim _{\eta \rightarrow 0^{+}} \mu\left(A_{i, \eta}\right)=\mu\left(S^{n-1} \cap\left(\xi_{i} \backslash \xi_{i-1}\right)\right) .
$$


To see this, first note that as $\eta$ decreases to 0 , the sets $A_{i, \eta} \cap \xi_{i}$ form an increasing family (with respect to set inclusion) whose union (over $\eta$ ) is $S^{n-1} \cap\left(\xi_{i} \backslash \xi_{i-1}\right)$. It follows that

$$
\liminf _{\eta \rightarrow 0^{+}} \mu\left(A_{i, \eta}\right) \geq \lim _{\eta \rightarrow 0^{+}} \mu\left(A_{i, \eta} \cap \xi_{i}\right)=\mu\left(S^{n-1} \cap\left(\xi_{i} \backslash \xi_{i-1}\right)\right) .
$$

Obviously, $A_{i, \eta} \subset B_{i, \eta}$. As $\eta$ is decreasing (to 0 ), the sets $B_{i, \eta}$ form a decreasing family (with respect to set inclusion) whose intersection (over $\eta$ ) is $S^{n-1} \cap\left(\xi_{i} \backslash \xi_{i-1}\right)$. Thus,

$$
\limsup _{\eta \rightarrow 0^{+}} \mu\left(A_{i, \eta}\right) \leq \lim _{\eta \rightarrow 0^{+}} \mu\left(B_{i, \eta}\right)=\mu\left(S^{n-1} \cap\left(\xi_{i} \backslash \xi_{i-1}\right)\right),
$$

which gives (6.7).

Combining (6.7) with (6.6) shows that we may choose $\eta_{o} \in(0,1 / \sqrt{n})$ small enough to satisfy

$$
\frac{1}{i} \sum_{j=1}^{i} \mu\left(A_{j, \eta_{o}}\right)<\frac{1}{n}, \quad \text { for all } i=1, \ldots, n-1 .
$$

But, since $\mu$ is a probability measure on $S^{n-1}$ and the $A_{j, \eta_{o}}$ form a disjoint partition of $S^{n-1}$, we have

$$
\sum_{j=1}^{n} \mu\left(A_{j, \eta_{o}}\right)=1
$$

Letting $\alpha_{j}=\mu\left(A_{j, \eta_{o}}\right)$, for $j=1, \ldots, n$, Lemma 6.1 yields a $t \in(0,1]$ such that for $\lambda=(1-t) / n$, and

$$
\beta_{i}=\alpha_{i}-\lambda, \text { for all } i=1, \ldots, n-1, \quad \text { while } \beta_{n}=\alpha_{n}-\lambda-t,
$$

we have

$$
\beta_{1}+\cdots+\beta_{i} \leq 0, \quad \text { for all } i=1, \ldots, n-1,
$$

and

$$
\beta_{1}+\cdots+\beta_{n}=0
$$

(Note that $\eta_{o}$ and $t$ are positive numbers independent of $l$.)

By (6.5), we have $\left|u_{i, l}-u_{i}\right|<\frac{\eta_{o}}{2}$, for all $i=1, \ldots, n$ and sufficiently large $l$. Then for $u \in A_{i, \eta_{o}}$,

$$
\begin{aligned}
\left|u \cdot u_{i, l}\right| & \geq\left|u \cdot u_{i}\right|-\left|u \cdot\left(u_{i, l}-u_{i}\right)\right| \\
& \geq\left|u \cdot u_{i}\right|-\left|u_{i, l}-u_{i}\right| \\
& \geq \frac{\eta_{o}}{2},
\end{aligned}
$$

for all $i=1, \ldots, n$. It follows from (6.4) that for $u \in A_{i, \eta_{o}}$,

$$
h_{Q_{l}}(u) \geq \frac{\eta_{o}}{2} h_{i, l},
$$

for all $i=1, \ldots, n$ and sufficiently large $l$.

It will be convenient to define $h_{n+1, l}=1$, for all $l$. For sufficiently large $l$, using the fact that the $A_{i, \eta_{o}}$ form a partition of $S^{n-1}$, followed by (6.11), again using the fact that the $A_{i, \eta_{o}}$ form a partition of $S^{n-1}$ and that $\mu$ is a probability measure, then using (6.8), then the fact that $\lambda$ is non-negative and the hypothesis 
that $h_{1, l} \cdots h_{n, l} \geq 1$, for all $l$, and finally from $0<h_{i, l} \leq h_{i+1, l}$, for $i=1, \ldots, n-1$, and (6.9) together with (6.10), we get

$$
\begin{aligned}
\int_{S^{n-1}} \log h_{Q_{l}} d \mu & =\sum_{i=1}^{n} \int_{A_{i, \eta_{o}}} \log h_{Q_{l}} d \mu \\
& \geq \log \frac{\eta_{o}}{2} \sum_{i=1}^{n} \mu\left(A_{i, \eta_{o}}\right)+\sum_{i=1}^{n} \mu\left(A_{i, \eta_{o}}\right) \log h_{i, l} \\
& =\log \frac{\eta_{o}}{2}+\sum_{i=1}^{n} \alpha_{i} \log h_{i, l} \\
& =\log \frac{\eta_{o}}{2}+t \log h_{n, l}+\sum_{i=1}^{n} \lambda \log h_{i, l}+\sum_{i=1}^{n} \beta_{i} \log h_{i, l} \\
& =\log \left(\frac{\eta_{o}}{2} h_{n, l}^{t}\right)+\lambda \log \left(h_{1, l} \cdots h_{n, l}\right)+\sum_{i=1}^{n} \beta_{i} \log h_{i, l} \\
& \geq \log \left(\frac{\eta_{o}}{2} h_{n, l}^{t}\right)+\sum_{i=1}^{n} \beta_{i} \log h_{i, l} \\
& =\log \left(\frac{\eta_{o}}{2} h_{n, l}^{t}\right)+\sum_{i=1}^{n}\left(\beta_{1}+\cdots+\beta_{i}\right)\left(\log h_{i, l}-\log h_{i+1, l}\right) \\
& \geq \log \left(\frac{\eta_{o}}{2} h_{n, l}^{t}\right) .
\end{aligned}
$$

Since $t>0$, and by hypothesis $\lim _{l \rightarrow \infty} h_{n, l}=\infty$, from (6.12) it follows that

$$
\lim _{l \rightarrow \infty} \int_{S^{n-1}} \log h_{Q_{l}} d \mu=\infty \text {. }
$$

Theorem 6.3. Suppose $n \geq 2$, and $\mu$ is an even finite Borel measure on $S^{n-1}$ that satisfies the strict subspace concentration inequality. Then there exists an originsymmetric convex body $K \in \mathcal{K}_{e}^{n}$ such that

$$
\inf \left\{\int_{S^{n-1}} \log h_{Q} d \mu: V(Q)=|\mu| \text { and } Q \in \mathcal{K}_{e}^{n}\right\}=\int_{S^{n-1}} \log h_{K} d \mu .
$$

Proof. Without loss of generality, assume that $\mu$ is a probability measure. Take a sequence $Q_{l} \in \mathcal{K}_{e}^{n}$ such that $V\left(Q_{l}\right)=1$ and

$$
\lim _{l \rightarrow \infty} \Phi_{\mu}\left(Q_{l}\right)=\inf \left\{\Phi_{\mu}(Q): V(Q)=1 \text { and } Q \in \mathcal{K}_{e}^{n}\right\} .
$$

Since the dilation, $\omega_{n}^{-1 / n} B$, of the unit ball has unit volume, and $\Phi_{\mu}\left(\omega_{n}^{-1 / n} B\right)=$ $-\frac{1}{n} \log \omega_{n}$, it follows that

$$
\lim _{l \rightarrow \infty} \Phi_{\mu}\left(Q_{l}\right) \leq-\frac{1}{n} \log \omega_{n}
$$

By John's theorem [32, there exists an ellipsoid $E_{l}$ centered at the origin such that

$$
E_{l} \subset Q_{l} \subset \sqrt{n} E_{l} .
$$

Let $u_{1, l}, \ldots, u_{n, l} \in S^{n-1}$ be the principal directions of $E_{l}$ indexed to satisfy

$$
h_{1, l} \leq \cdots \leq h_{n, l}, \quad \text { where } h_{i, l}=h_{E_{l}}\left(u_{i, l}\right), \quad \text { for } i=1, \ldots, n .
$$


Next we define the cross-polytope

$$
C_{l}=\left[ \pm h_{1, l} u_{1, l}, \ldots, \pm h_{n, l} u_{n, l}\right] .
$$

Since $C_{l} \subset E_{l} \subset \sqrt{n} C_{l}$, we have

$$
C_{l} \subset Q_{l} \subset n C_{l} .
$$

We deduce from $V\left(Q_{l}\right)=1$ that $V\left(C_{l}\right) \geq n^{-n}$. Thus,

$$
\prod_{i=1}^{n} h_{i, l}=\frac{n ! V\left(C_{l}\right)}{2^{n}} \geq \gamma,
$$

where $\gamma=\frac{n !}{2^{n} n^{n}}$.

Suppose that the sequence $\left\{Q_{l}\right\}$ is not bounded. Then $\left\{C_{l}\right\}$ is not bounded, and thus, for a subsequence,

$$
\lim _{l \rightarrow \infty} h_{n, l}=\infty .
$$

In view of (6.14) and (6.15), applying Lemma 6.2 to $C_{l}^{\prime}=\gamma^{\frac{-1}{n}} C_{l}$ yields that $\left\{\Phi_{\mu}\left(C_{l}^{\prime}\right)\right\}$ is not bounded from above. Thus, $\left\{\Phi_{\mu}\left(Q_{l}\right)\right\}$ is not bounded from above. This contradicts (6.13). Therefore, the sequence $\left\{Q_{l}\right\}$ is bounded. By the Blaschke selection theorem, $\left\{Q_{l}\right\}$ has a subsequence that converges to an origin-symmetric convex body $K$. Thus, the minimization problem (4.2) attains its minimum at $K$.

\section{Existence For the EVEn LOGARIthmic Minkowski PROBlem}

Here we prove the sufficiency part of Theorem 1.1

Lemma 7.1. Suppose $n \geq 2$ and $\mu$ is a finite Borel measure on $S^{n-1}$ that satisfies the subspace concentration condition. If $\xi$ is a subspace of $\mathbb{R}^{n}$ for which there is equality in (1.4), that is,

$$
\mu\left(\xi \cap S^{n-1}\right)=\frac{1}{n} \mu\left(S^{n-1}\right) \operatorname{dim} \xi,
$$

then $\mu$ restricted to $S^{n-1} \cap \xi$ satisfies the subspace concentration condition.

Proof. Let $m=\operatorname{dim} \xi$, and let $S^{m-1}=\xi \cap S^{n-1}$. Then (7.1) states that

$$
\frac{1}{m} \mu\left(S^{m-1}\right)=\frac{1}{n} \mu\left(S^{n-1}\right) \text {. }
$$

Suppose $\xi_{k} \subset \xi$ is a $k$-dimensional subspace. Then, from the definition of $S^{m-1}$, the fact that $\xi_{k} \subset \xi$, the fact that $\mu$ satisfies the subspace concentration condition, and finally (7.2), we have:

$$
\begin{aligned}
\mu\left(\xi_{k} \cap S^{m-1}\right) & =\mu\left(\xi_{k} \cap\left(\xi \cap S^{n-1}\right)\right) \\
& =\mu\left(\xi_{k} \cap S^{n-1}\right) \\
& \leq \frac{k}{n} \mu\left(S^{n-1}\right) \\
& =\frac{1}{m} \mu\left(S^{m-1}\right) \operatorname{dim} \xi_{k} .
\end{aligned}
$$

This not only establishes the subspace concentration inequality for $\mu$ restricted to $\xi \cap S^{n-1}$, but shows that equality in

$$
\mu\left(\xi_{k} \cap S^{m-1}\right) \leq \frac{1}{m} \mu\left(S^{m-1}\right) \operatorname{dim} \xi_{k}
$$


implies that

$$
\mu\left(\xi_{k} \cap S^{n-1}\right)=\frac{k}{n} \mu\left(S^{n-1}\right) .
$$

But (7.3) and the fact that $\mu$ satisfies the subspace concentration condition yield the existence of a subspace $\xi_{n-k}$ complementary to $\xi_{k}$ in $\mathbb{R}^{n}$ such that $\mu$ is concentrated on $S^{n-1} \cap\left(\xi_{k} \cup \xi_{n-k}\right)$. This implies that $\mu$ restricted to $\xi \cap S^{n-1}=S^{m-1}$ is concentrated on $S^{m-1} \cap\left(\xi_{k} \cup\left(\xi_{n-k} \cap \xi\right)\right)$.

If $\mu$ is a Borel measure on $S^{n-1}$ and $\xi$ is a proper subspace of $\mathbb{R}^{n}$, it will be convenient to write $\mu_{\xi}$ for the restriction of $\mu$ to $S^{n-1} \cap \xi$.

Lemma 7.2. Let $\xi$ and $\xi^{\prime}$ be complementary subspaces in $\mathbb{R}^{n}$ with $0<\operatorname{dim} \xi=$ $m<n$. Suppose $\mu$ is an even Borel measure on $S^{n-1}$ that is concentrated on $S^{n-1} \cap\left(\xi \cup \xi^{\prime}\right)$, and so that

$$
\mu\left(\xi \cap S^{n-1}\right)=\frac{m}{n} \mu\left(S^{n-1}\right) .
$$

If $\mu_{\xi}$ and $\mu_{\xi^{\prime}}$ are cone-volume measures of convex bodies in the spaces $\xi$ and $\xi^{\prime}$, then $\mu$ is the cone-volume measure of a convex body in $\mathbb{R}^{n}$.

Proof. By hypothesis there exist convex bodies $C$ in $\xi$ and $C^{\prime}$ in $\xi^{\prime}$ so that

$$
V_{C}=a \mu_{\xi}, \quad V_{C^{\prime}}=a \mu_{\xi^{\prime}},
$$

where the positive constant $a$ will be chosen appropriately later. Construct convex bodies $D$ in $\xi^{\prime}$ and $D^{\prime}$ in $\xi^{\perp}$ by

$$
\begin{aligned}
D & =\left\{\left(x+\xi^{\perp}\right) \cap \xi^{\perp}: x \in C\right\}, \\
D^{\prime} & =\left\{\left(x+\xi^{\perp}\right) \cap \xi^{\perp}: x \in C^{\prime}\right\} .
\end{aligned}
$$

Then $\mathrm{P}_{\xi} D=C$ and $\mathrm{P}_{\xi^{\prime}} D^{\prime}=C^{\prime}$. Now,

$$
\frac{V_{m}(D)}{V_{m}(C)}=\frac{V_{n-m}\left(D^{\prime}\right)}{V_{n-m}\left(C^{\prime}\right)}=r
$$

where $r$ can be viewed as the reciprocal of the cosine of the angle between $\xi$ and $\xi^{\prime \perp}$. Then

$$
\begin{aligned}
V_{m}(D) & =r V_{m}(C)=r V_{C}\left(S^{n-1} \cap \xi\right) \\
& =r a \mu_{\xi}\left(S^{n-1} \cap \xi\right)=\operatorname{ra\mu }\left(S^{n-1} \cap \xi\right)=r a \frac{m}{n} \mu\left(S^{n-1}\right) .
\end{aligned}
$$

Similarly,

Observe that

$$
V_{n-m}\left(D^{\prime}\right)=r a \frac{n-m}{n} \mu\left(S^{n-1}\right)
$$

$$
\partial\left(D+D^{\prime}\right)=\left(\partial D+\operatorname{relint} D^{\prime}\right) \cup\left(\operatorname{relint} D+\partial D^{\prime}\right) \cup\left(\partial D+\partial D^{\prime}\right) .
$$

Consider $\mathbb{R}^{n}$ as the orthogonal sum of $\xi$ and $\xi^{\perp}$. Write $y=\left(y_{1}, y_{2}\right) \in \mathbb{R}^{n}$ and identify $y_{1}$ with $\left(y_{1}, 0\right)$ and $y_{2}$ with $\left(0, y_{2}\right)$. Obviously for each $y=\left(y_{1}, y_{2}\right) \in \partial D$, we have $y_{1} \in \partial C$ and $y=\left(y_{1}+\xi^{\perp}\right) \cap \xi^{\prime \perp}$. For the outer normals on $\partial D+\operatorname{relint} D^{\prime}$, we have $\nu_{D+D^{\prime}}\left(y+y^{\prime}\right)=\nu_{D+D^{\prime}}(y)$. These normals are orthogonal to $\xi^{\perp} \supset D^{\prime}$ and thus belong to $S^{n-1} \cap \xi$. The normal $\nu_{D+D^{\prime}}(y)$ is also orthogonal to the $(m-1)$ dimensional support plane of $D$ at $y \in \partial D$ in $\xi^{\prime \perp}$ and thus is orthogonal to the orthogonal projection of the support plane of $D$ at $y$ onto $\xi$, that is, orthogonal to the $(m-1)$-dimensional support plane of $C$ at $y_{1} \in \partial C$ in $\xi$. It follows that 
$\nu_{D+D^{\prime}}(y)=\nu_{C}\left(y_{1}\right)$. We now see that for $\mathcal{H}^{m-1}$-almost all $y \in \partial D$ and for all $y^{\prime} \in \operatorname{relint} D^{\prime}$,

$$
\nu_{D+D^{\prime}}\left(y+y^{\prime}\right)=\nu_{C}\left(y_{1}\right) \text { and } y \cdot \nu_{C}\left(y_{1}\right)=y_{1} \cdot \nu_{C}\left(y_{1}\right),
$$

for $\mathcal{H}^{m-1}$-almost all $y_{1} \in \partial C$.

Suppose $\omega \subset S^{n-1} \cap \xi$. Obviously,

$$
\nu_{D+D^{\prime}}^{-1}(\omega) \subset \partial D+\operatorname{relint} D^{\prime} .
$$

From the definition of a cone-volume measure, (7.5), and using the fact that $\nu_{C}^{-1}(\omega)$ is the orthogonal projection of $\nu_{D+D^{\prime}}^{-1}(\omega)$ onto $\xi$, we have

$$
\begin{aligned}
V_{D+D^{\prime}}(\omega) & =\frac{1}{n} \int_{\nu_{D+D^{\prime}}^{-1}(\omega)}\left(y+y^{\prime}\right) \cdot \nu_{D+D^{\prime}}\left(y+y^{\prime}\right) d \mathcal{H}^{n-1}\left(y+y^{\prime}\right) \\
& =\frac{1}{n} \int_{\nu_{D+D^{\prime}}^{-1}(\omega)} y \cdot \nu_{C}\left(y_{1}\right) d \mathcal{H}^{n-1}\left(y+y^{\prime}\right) \\
& =\frac{1}{n} \int_{\nu_{C}^{-1}(\omega)} y_{1} \cdot \nu_{C}\left(y_{1}\right) d \mathcal{H}^{m-1}\left(y_{1}\right) V_{n-m}\left(D^{\prime}\right) \\
& =\frac{m}{n} V_{n-m}\left(D^{\prime}\right) V_{C}(\omega) \\
& =\frac{m(n-m)}{n^{2}} r a^{2} \mu\left(S^{n-1}\right) \mu_{\xi}(\omega) .
\end{aligned}
$$

Similarly, for $\omega^{\prime} \subset S^{n-1} \cap \xi^{\prime}$,

$$
V_{D+D^{\prime}}\left(\omega^{\prime}\right)=\frac{m(n-m)}{n^{2}} r a^{2} \mu\left(S^{n-1}\right) \mu_{\xi^{\prime}}\left(\omega^{\prime}\right) .
$$

Now choose $a$ so that

$$
\frac{m(n-m)}{n^{2}} r a^{2} \mu\left(S^{n-1}\right)=1
$$

Then

$$
\begin{aligned}
V_{D+D^{\prime}}(\omega) & =\mu_{\xi}(\omega), \quad \text { for } \omega \subset S^{n-1} \cap \xi, \\
V_{D+D^{\prime}}\left(\omega^{\prime}\right) & =\mu_{\xi^{\prime}}\left(\omega^{\prime}\right), \quad \text { for } \omega^{\prime} \subset S^{n-1} \cap \xi^{\prime} .
\end{aligned}
$$

Using (7.4) we see that the surface area measure $S_{D+D^{\prime}}$ of $D+D^{\prime}$ is concentrated on $S^{n-1} \cap\left(\xi \cup \xi^{\prime}\right)$, and thus the cone-volume measure $V_{D+D^{\prime}}$ is as well. From this and the fact that $\mu$ is concentrated on $S^{n-1} \cap\left(\xi \cup \xi^{\prime}\right)$, we conclude that

$$
V_{D+D^{\prime}}=\mu \text {. }
$$

Theorem 7.3. Suppose $n \geq 1$ and $\mu$ is a non-zero even finite Borel measure on $S^{n-1}$ that satisfies the subspace concentration condition. Then $\mu$ is the cone-volume measure of an origin-symmetric convex body in $\mathbb{R}^{n}$.

Proof. We first assume that strict inequality holds in (1.4) for every linear subspace $\xi$ such that $0<\operatorname{dim} \xi<n$. By Theorem 6.3, the minimization problem (4.2) has a solution. Thus, by Lemma 4.1, the measure $\mu$ is a cone-volume measure of an origin-symmetric convex body in $\mathbb{R}^{n}$. 
Now suppose that equality holds in (1.4), i.e., suppose

$$
\mu\left(\xi \cap S^{n-1}\right)=\frac{1}{n} \mu\left(S^{n-1}\right) \operatorname{dim} \xi,
$$

for some linear subspace $\xi$ with $0<\operatorname{dim} \xi<n$. In this case, from the definition of a measure that satisfies the subspace concentration condition, there exists a linear subspace $\xi^{\prime}$ complementary to $\xi$ (obviously of dimension $n-\operatorname{dim} \xi$ ) such that

$$
\mu\left(\xi^{\prime} \cap S^{n-1}\right)=\frac{1}{n} \mu\left(S^{n-1}\right) \operatorname{dim} \xi^{\prime} .
$$

If it is not the case that strict inequality holds in (1.4) for every linear subspace $\xi$ such that $0<\operatorname{dim} \xi<n$, then we proceed by induction on the dimension of the ambient space. We start with $n=2$. In this case we must have $\operatorname{dim} \xi=1$ and $\mu\left(\xi \cap S^{1}\right)=\mu\left(S^{1}\right) / 2$. If we write $\xi \cap S^{1}=\left\{u_{0},-u_{0}\right\}$ and recall that $\mu$ is even, we see that $\mu\left(\left\{u_{0}\right\}\right)=\mu\left(\left\{-u_{0}\right\}\right)=\mu\left(S^{1}\right) / 4$. Since $\operatorname{dim} \xi=1$, we have $\operatorname{dim} \xi^{\prime}=1$, and if we write $\xi^{\prime} \cap S^{1}=\left\{u_{0}^{\prime},-u_{0}^{\prime}\right\}$, and recall that $\mu$ is even, it follows that $\mu\left(\left\{u_{0}^{\prime}\right\}\right)=\mu\left(\left\{-u_{0}^{\prime}\right\}\right)=\mu\left(S^{1}\right) / 4$. Thus the parallelogram (centered at the origin) whose sides are perpendicular to $u_{0}, u_{0}^{\prime}$ and whose area is $\mu\left(S^{1}\right)$ is the desired convex body in $\mathbb{R}^{2}$.

We now assume that $n \geq 3$ and that the existence has been established for all dimensions less than $n$. From Lemma 7.1, applied to the situations described by (7.6) and (7.7), it follows that the restrictions of $\mu$ to $\xi \cap S^{n-1}$ and to $\xi^{\prime} \cap S^{n-1}$ satisfy the subspace concentration conditions in $\xi$ and $\xi^{\prime}$, respectively. Therefore, there exist an origin-symmetric $m$-dimensional convex body $C$ in $\xi$ and an originsymmetric $(n-m)$-dimensional convex body $C^{\prime}$ in $\xi^{\prime}$ such that the restrictions of $\mu$ to $\xi \cap S^{n-1}$ and to $\xi^{\prime} \cap S^{n-1}$ are cone-volume measures of $C$ and $C^{\prime}$, respectively. Lemma 7.2 will now yield the desired result.

\section{ACKNOWLEDGEMENTS}

The authors thank the referees and Professor Rolf Schneider for their very helpful comments.

\section{REFERENCES}

[1] A.D. Alexandrov, Existence and uniqueness of a convex surface with a given integral curvature, C. R. (Doklady) Acad. Sci. USSR (N.S.) 35 (1942), 131-134. MR0007625 (4:169c)

[2] B. Andrews, Gauss curvature flow: the fate of the rolling stones, Invent. Math. 138 (1999), 151-161. MR1714339 (2000i:53097)

[3] B. Andrews, Classification of limiting shapes for isotropic curve flows, J. Amer. Math. Soc. 16 (2003), 443-459. MR.1949167 (2004a:53083)

[4] F. Barthe, O. Guedon, S. Mendelson, and A. Naor, A probabilistic approach to the geometry of the $l_{p}^{n}$-ball, Ann. of Probability 33 (2005), 480-513. MR2123199 (2006g:46014)

[5] L. Caffarelli, Interior $W^{2, p}$-estimates for solutions of the Monge-Ampère equation, Ann. of Math. (2) 131 (1990), 135-150. MR1038360 (91f:35059)

[6] S. Campi and P. Gronchi, The $L^{p}$-Busemann-Petty centroid inequality, Adv. Math. 167 (2002), 128-141. MR1901248 (2003e:52011) 
[7] W. Chen, $L_{p}$ Minkowski problem with not necessarily positive data, Adv. Math. 201 (2006), 77-89. MR2204749 (2007b:34085)

[8] S.-Y. Cheng and S.-T. Yau, On the regularity of the solution of the $n$-dimensional Minkowski problem, Comm. Pure Appl. Math. 29 (1976), 495-516. MR0423267 (54:11247)

[9] K.-S. Chou and X.-J. Wang, The $L_{p}$-Minkowski problem and the Minkowski problem in centroaffine geometry, Adv. Math. 205 (2006), 33-83. MR2254308 (2007f:52019)

[10] B. Chow, Deforming convex hypersurfaces by the nth root of the Gaussian curvature, J. Differential Geom. 22 (1985), 117-138. MR826427 (87f:58155)

[11] A. Cianchi, E. Lutwak, D. Yang, and G. Zhang, Affine Moser-Trudinger and Morrey-Sobolev inequalities, Calc. Var. Partial Differential Equations 36 (2009), 419-436. MR2551138 (2010h:46041)

[12] A. Colesanti and M. Fimiani, The Minkowski problem for torsional rigidity, Indiana. Univ. Math. J. 59 (2010), 1013-1039. MR2779070(2012d:52007)

[13] W. Firey, Shapes of worn stones, Mathematika 21 (1974), 1-11. MR0362045 (50:14487)

[14] M. Gage and R. Hamilton, The heat equation shrinking convex plane curves, J. Differential Geom. 23 (1986), 69-96. MR840401 (87m:53003)

[15] R.J. Gardner, Geometric Tomography, 2nd edition, Encyclopedia of Mathematics and its Applications, Cambridge University Press, Cambridge, 2006. MR2251886 (2007i:52010)

[16] R.J. Gardner, The Brunn-Minkowski inequality, Bull. Amer. Math. Soc. 39 (2002), 355-405. MR.1898210 (2003f:26035)

[17] R.J. Gardner and G. Zhang, Affine inequalities and radial mean bodies, Amer. J. Math. 120 (1998), 505-528. MR1623396(99e:52006)

[18] M. Gromov and V.D. Milman, Generalization of the spherical isoperimetric inequality for uniformly convex Banach Spaces, Composito Math. 62 (1987), 263-282. MR901393 (89f:46031)

[19] P.M. Gruber, Convex and discrete geometry, Grundlehren der Mathematischen Wissenschaften, 336. Springer, Berlin, 2007. MR2335496 (2008f:52001)

[20] B. Guan and P. Guan, Convex hypersurfaces of prescribed curvatures, Ann. of Math. (2) 156 (2002), 655-673. MR1933079 (2003i:53046)

[21] P. Guan and C.-S. Lin, On equation $\operatorname{det}\left(u_{i j}+\delta_{i j} u\right)=u^{p} f$ on $S^{n}$, preprint No. 2000-7, NCTS in Tsing-Hua University, 2000.

[22] P. Guan and X. Ma, The Christoffel-Minkowski problem I: Convexity of solutions of a Hessian equation, Invent. Math. 151 (2003), 553-577. MR1961338(2004a:35071)

[23] C. Haberl, E. Lutwak, D. Yang, and G. Zhang, The even Orlicz Minkowski problem, Adv. Math. 224 (2010), 2485-2510. MR 2652213(2011k:52006)

[24] C. Haberl and F. Schuster, General $L_{p}$ affine isoperimetric inequalities, J. Differential Geom. 83 (2009), 1-26. MR2545028 (2010j:52026)

[25] C. Haberl and F. Schuster, Asymmetric affine $L_{p}$ Sobolev inequalities, J. Funct. Anal. 257 (2009), 641-658. MR2530600 (2010j:46068)

[26] C. Haberl, F. Schuster, and J. Xiao, An asymmetric affine Pólya-Szegő principle, Math. Ann. 352 (2012), 517-542.

[27] B. He, G. Leng, and K. Li, Projection problems for symmetric polytopes, Adv. Math. 207 (2006), 73-90. MR2264066 (2007k:52018)

[28] C. Hu, X. Ma, and C. Shen, On the Christoffel-Minkowski problem of Firey's p-sum, Calc. Var. Partial Differential Equations 21 (2004), 137-155. MR2085300 (2005g:52019)

[29] D. Hug, E. Lutwak, D. Yang, and G. Zhang, On the $L_{p}$ Minkowski problem for polytopes, Discrete Comput. Geom. 33 (2005), 699-715. MR2132298 (2006b:52005)

[30] D. Jerison, A Minkowski problem for electrostatic capacity, Acta Math. 176 (1996), 1-47. MR.1395668 (97e:31003)

[31] M. Y. Jiang, Remarks on the 2-dimensional $L_{p}$-Minkowski problem, Adv. Nonlinear Stud. 10 (2010), 297-313. MR2656684 (2011e:34096)

[32] F. John, Polar correspondence with respect to a convex region, Duke Math. J. 3 (1937), 355-369. MR 1545993

[33] D. Klain, The Minkowski problem for polytopes, Adv. Math. 185 (2004), 270-288. MR2060470 (2005b:52013)

[34] H. Lewy, On differential geometry in the large. I. Minkowski's problem, Trans. Amer. Math. Soc. 43 (1938), 258-270. MR.1501942 
[35] M. Ludwig, Ellipsoids and matrix-valued valuations, Duke Math. J. 119 (2003), 159-188. MR.1991649 (2004e:52015)

[36] M. Ludwig, General affine surface areas, Adv. Math. 224 (2010), 2346-2360. MR2652209 (2011h:52006)

[37] M. Ludwig and M. Reitzner, A classification of SL(n) invariant valuations, Ann. of Math. (2) 172 (2010), 1219-1267. MR2680490 (2011g:52025)

[38] E. Lutwak, The Brunn-Minkowski-Firey theory. I. Mixed volumes and the Minkowski problem, J. Differential Geom. 38 (1993), 131-150. MR.1231704 (94g:52008)

[39] E. Lutwak and V. Oliker, On the regularity of solutions to a generalization of the Minkowski problem, J. Differential Geom. 41 (1995), 227-246. MR.1316557 (95m:52007)

[40] E. Lutwak, D. Yang, and G. Zhang, $L_{p}$ affine isoperimetric inequalities, J. Differential Geom. 56 (2000), 111-132. MR.1863023 (2002h:52011)

[41] E. Lutwak, D. Yang, and G. Zhang, A new ellipsoid associated with convex bodies, Duke Math. J. 104 (2000), 375-390. MR1781476 (2001j:52011)

[42] E. Lutwak, D. Yang, and G. Zhang, The Cramer-Rao inequality for star bodies, Duke Math. J. 112 (2002), 59-81. MR.1890647(2003f:52006)

[43] E. Lutwak, D. Yang, and G. Zhang, Sharp affine $L_{p}$ Sobolev inequalities, J. Differential Geom. 62 (2002), 17-38. MR.1987375 (2004d:46039)

[44] E. Lutwak, D. Yang, and G. Zhang, On the $L_{p}$-Minkowski problem, Trans. Amer. Math. Soc. 356 (2004), no. 11, 4359-4370. MR2067123 (2005d:52013)

[45] E. Lutwak, D. Yang, and G. Zhang, Volume inequalities for subspaces of $L_{p}$, J. Differential Geom. 68 (2004), 159-184. MR2152912 (2006j:52010)

[46] E. Lutwak, D. Yang, and G. Zhang, Optimal Sobolev norms and the $L^{p}$ Minkowski problem, Int. Math. Res. Not. 2006, Art. ID 62987, 21 pp. MR.2211138(2007d:52007)

[47] E. Lutwak and G. Zhang, Blaschke-Santaló inequalities, J. Differential Geom. 47 (1997), 1-16. MR1601426(2000c:52011)

[48] H. Minkowski, Allgemeine Lehrsätze über die convexen Polyeder, Nachr. Ges. Wiss. Göttingen, 198-219. Gesammelte Abhandlungen, Vol. II, Teubner, Leipzig, 1911, pp. 103-121.

[49] A. Naor, The surface measure and cone measure on the sphere of $l_{p}^{n}$, Trans. Amer. Math. Soc. 359 (2007), 1045-1079. MR2262841 (2007g:60006)

[50] A. Naor and D. Romik, Projecting the surface measure of the sphere of $l_{p}^{n}$, Ann. Inst. H. Poincaré Probab. Statist. 39 (2003), 241-261. MR.1962135 (2004d:60063)

[51] L. Nirenberg, The Weyl and Minkowski problems in differential geometry in the large, Comm. Pure and Appl. Math. 6 (1953), 337-394. MR0058265 (15:347b)

[52] G. Paouris, Concentration of mass on convex bodies, Geom. Funct. Anal. 16 (2006), 10211049. MR2276533 (2007k:52009)

[53] G. Paouris and E. Werner, Relative entropy of cone measures and $L_{p}$ centroid bodies, Proc. London Math. Soc. 104 (2012), 253-286.

[54] A.V. Pogorelov, The Minkowski multidimensional problem, V.H. Winston \& Sons, Washington, D.C., 1978. MR0478079 (57:17572)

[55] R. Schneider, Convex bodies: the Brunn-Minkowski theory, Encyclopedia of Mathematics and its Applications, Cambridge University Press, Cambridge, 1993. MR.1216521 (94d:52007)

[56] A. Stancu, The discrete planar L0-Minkowski problem, Adv. Math. 167 (2002), 160-174. MR.1901250 (2003e:52010)

[57] A. Stancu, On the number of solutions to the discrete two-dimensional $L_{0}$-Minkowski problem, Adv. Math. 180 (2003), 290-323. MR2019226 (2004k:52003)

[58] A. Stancu, Centro-affine invariants for smooth convex bodies, Int. Math. Res. Not. 2012, 2289-2320.

[59] A. C. Thompson, Minkowski geometry, Encyclopedia of Mathematics and its Applications, Cambridge University Press, Cambridge, 1996. MR.1406315 (97f:52001)

[60] Kaising Tso, Deforming a hypersurface by its Gauss-Kronecker curvature, Comm. Pure Appl. Math. 38 (1985), 867-882. MR812353 (87e:53009)

[61] V. Umanskiy, On solvability of two-dimensional $L_{p}$-Minkowski problem, Adv. Math. 180 (2003), 176-186. MR2019221 (2004i:53101)

[62] G. Xiong, Extremum problems for the cone volume functional for convex polytopes, Adv. Math. 225 (2010), 3214-3228. MR2729006 (2012b:52018)

[63] G. Zhang, The affine Sobolev inequality, J. Differential Geom. 53 (1999), 183-202. MR:1776095 (2001m:53136) 
852 KÁROLY J. BÖRÖCZKY, ERWIN LUTWAK, DEANE YANG, AND GAOYONG ZHANG

Alfréd Rényi Institute of Mathematics, Hungarian Academy of Sciences

E-mail address: carlos@renyi.hu

Department of Mathematics, Polytechnic Institute of New York University, BrookLYN, NEW YORK 11201

E-mail address: elutwak@poly.edu

Department of Mathematics, Polytechnic Institute of New York University, BrookLYN, NEW YORK 11201

E-mail address: dyang@poly.edu

Department of Mathematics, Polytechnic Institute of New York University, BrookLYN, NEW YORK 11201

E-mail address: gzhang@poly.edu 